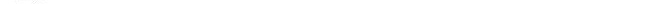

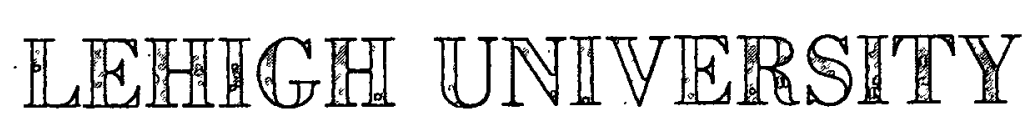

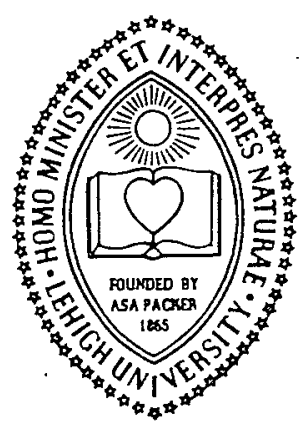

TWO COLLINEAR CRACKS IN A CYLINDRICAL SHELL

BY

F. ERDOGAN

M. RATWANI

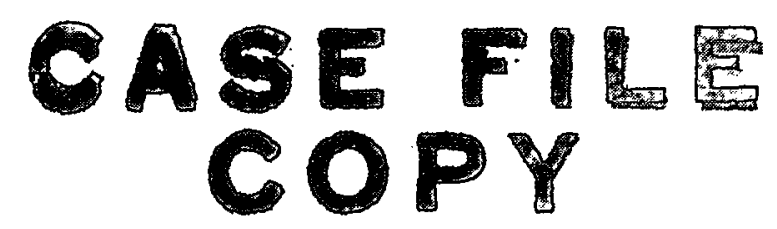

TECHNICAL REPORT NASA TR-73-3

FEBRUARY 1973

National Aeronautics and Space Administration 


\section{A NOTE ON THE INTERFERENCE OF TWO \\ COLLINEAR CRACKS IN A CYLINDRICAL SHELL*}

by

F. Erdogan and M. Ratwani

Lehigh University, Bethlehem, Pa.

\section{INTRODUCTION}

Due to its applications to a wide variety of structural problems of practical importance, in recent years there has been a considerable interest in the problem of shallow shells containing a through crack. An up-to-date survey of the problem and a complete list of references were given in a recent paper [1]. Without any exception, in all studies which appeared in the literature it was assumed that the shell contains a single meridional crack. On the other hand, from the analysis of plane problems it is known that there is a strong interference between the stress fields around two cracks when the distance between the cracks decreases (see, e.g., [2]). Since the shell problem has somewhat peculiar aspects of its own, one may have to be cautious in attempting to predict the interference of crack stress fields in shells from that of flat plates. Therefore, it is worthwhile to look into a simple interference problem in shells and to compare the results with that of flat plates.

*This work was supported by The National Aeronautics and Space Administration under The Grant NGR-39-007-011. 


\section{THE STATEMENT OF THE PROBLEM AND THE RESULTS}

Consider the problem of a pressurized "shallow" cylindrical shell containing two collinear axial through cracks of equal lengths (see the insert in Figure 1). Let $2 \mathrm{a}$ be 1 ength and $2 \mathrm{c}$ be the center-to-center distance of the cracks. The external load is assumed to be the internal pressure $p_{0}$. Thus, the problem has a symmetry with respect to $x$ and $y$ axes in the tangent plane and, after reducing it to a perturbation problem through a proper superposition, it can be solved by expressing the unknown functions $F$ (the stress function) and $w$ (the $z$-displacement) in terms of Fourier integrals. The procedure to be followed in the solution would be identical to that of a single crack. The problem may again be reduced to a pair of singular integral equations similar to that of a single crack - the only difference being in the Fredholm kernels. Hence, in this note we will omit the analytical details and discuss only the results.

Let the equivalent stress intensity factor in flat plate be defined as

$$
k_{p}=\frac{p_{0}^{R}}{h} \sqrt{a}
$$

where $p_{0}$ is the internal pressure, $R$ is the mean radius of curvature, $h$ is the thickness and $a$ is the half-crack length in the cylindrical shel1. The computed results shown in Figures 1 and 2 give the shell-to-flat plate stress intensity factor ratios which are defined by

$$
A_{m}=\frac{k_{m}^{s}}{k_{p}}, \quad A_{b}=\frac{k_{b}^{s}}{k_{p}},
$$


where $k_{m}^{S}$ and $k_{b}^{S}$ are, respectively, the membrane and bending components of the stress intensity factor in the shell. The superscripts $i$ and $O$ on $A_{m}$ and $A_{b}$ refer to the inner and outer crack tips, respectively. The figures show the results for $\lambda=1,2,3$, where the shell parameter $\lambda$ is given by ${ }^{*}$

$$
\lambda=\left[12\left(1-\nu^{2}\right)\right]^{\frac{1}{4}} \frac{\mathrm{a}}{\sqrt{\mathrm{Rh}}} \text {. }
$$

Figure 1 also shows the stress intensity factors for a flat plate (i.e., for $\lambda=0$ ) calculated from the expressions given in [2], i.e.,

$$
\begin{aligned}
& \frac{k^{i}}{\sigma_{0} \sqrt{a}}=\frac{b_{1}{ }^{2} E(m) / k(m)-a_{1}{ }^{2}}{\left(b_{1}-a_{1}\right) \sqrt{a_{1}\left(b_{1}+a_{1}\right) / 2}}, \\
& \frac{k^{0}}{\sigma_{0} \sqrt{a}}=\frac{b_{1}{ }^{2}[1-E(m) / k(m)]}{\left(b_{1}-a_{1}\right) \sqrt{b_{1}\left(b_{1}+a_{1}\right) / 2}},
\end{aligned}
$$

where

$$
\sigma_{0}=p_{0} R / h, \quad a_{1}=c-a, \quad b_{1}=c+a, \quad m=1-\frac{a_{1}{ }^{2}}{b_{1}{ }^{2}},
$$

and $K(m)$ and $E(m)$ are the complete elliptic integrals of the first and the second kind, respectively.

For $a / c=0$ there is no interference between the stress fields of the two cracks and the results correspond to that obtained from the single crack solution. As $a / c \rightarrow 1$, i.e., as the size of the net ligament between the two cracks approaches zero, as expected, the stress intensity factors at the inner

\footnotetext{
*Since the Poisson's ratio $v$ also appears elsewhere in the analysis, in this example $v$ was assumed to be $1 / 3$.
} 
crack tips go to infinity and those at the outer tips approach the values corresponding to a single crack with twice the length. However, the results show some unexpected behavior for larger values of $\lambda$ and for approximately a/c $>0.4$. Figure 1, for example, shows that for $\lambda=2$ and $\lambda=3$, as a/c increases (i.e.. as the distance between the two cracks of fixed length decreases) there is first a reduction in the stress intensity factors before they begin to increase at a high rate as a/c approaches 1 . A partial explanation of this somewhat anomalous behavior may lie in the distribution of the displacement component $w$ normal to the shell surface. In a pressurized shell with a single crack it was shown that [3], although in the neighborhood of the crack there is an outward bulging in the shell, further away from the crack tips w changes sign and there is a zone depression. When the stress and displacement fields of the two cracks interfere, this depression may cause a reduction in the stress intensity factors.

\section{REFERENCES}

1. Erdogan, F: and Ratwani, M., "Fracture of Cylindrical and Spherical Shells Containing a Crack", Nuclear Engineering and Design, Vol. 20, 1972, pp. 265-286.

2. Erdogan, F., "On the Stress Distribution in Plates with Collinear Cuts under Arbitrary Loads", Proceedings the 4th U.S. National Congress of Applied Mechanics, ASME, Vol. 1 , 1962 , pp. 547-553.

3. Erdogan, F. and Ratwani, M., "Plasticity and the Crack Opening Displacement in Shells", International Journal of Fracture Mechanics, Vol. 8, 1972, pp. 413-426. 


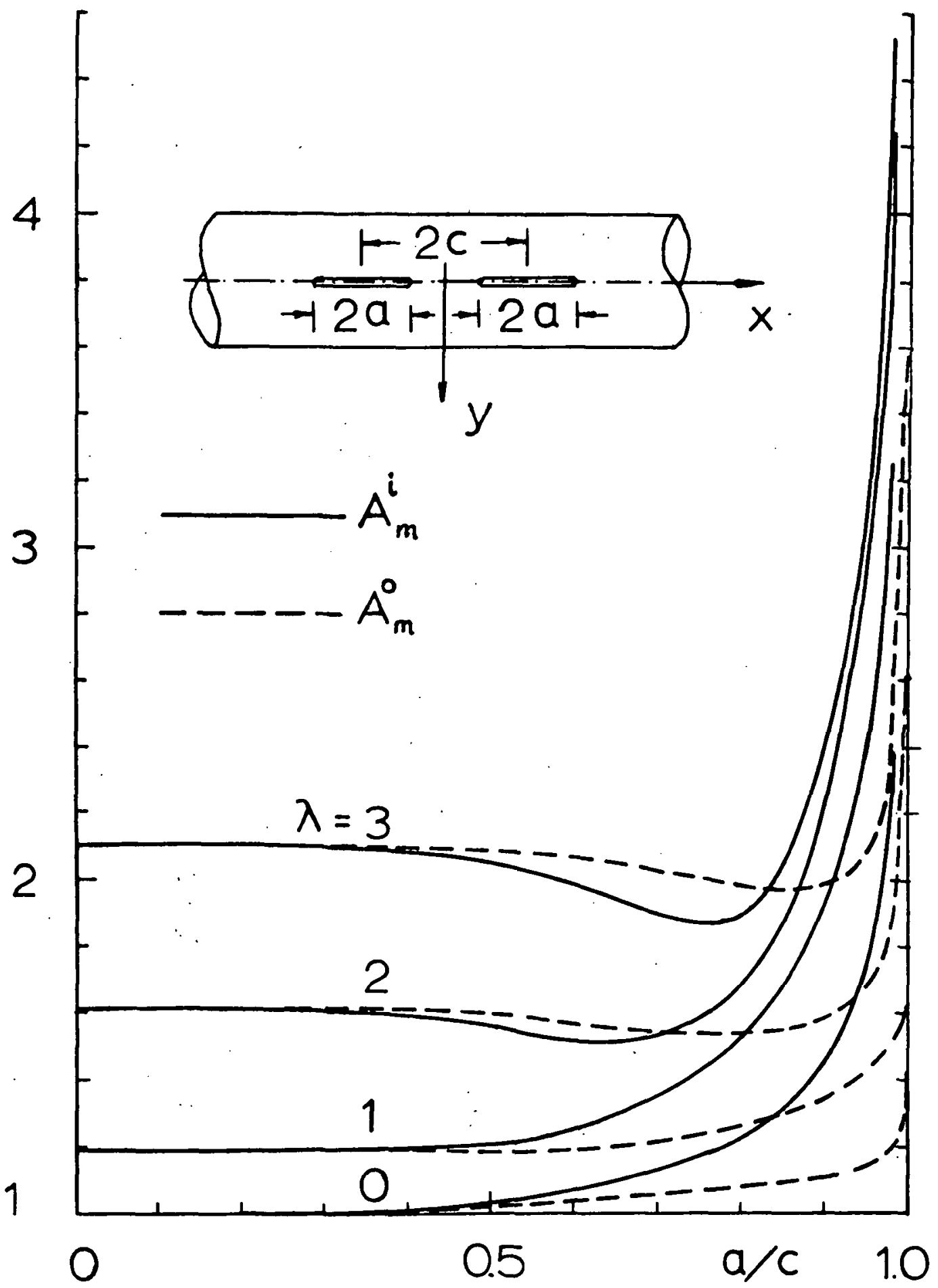

Figure 1. The membrane components of the stress intensity factor ratio in a pressurized cylindrical shell with two collinear cracks, $A_{m}^{i}$ for the inner crack tip and $A_{m}^{0}$ for the outer crack tip. 


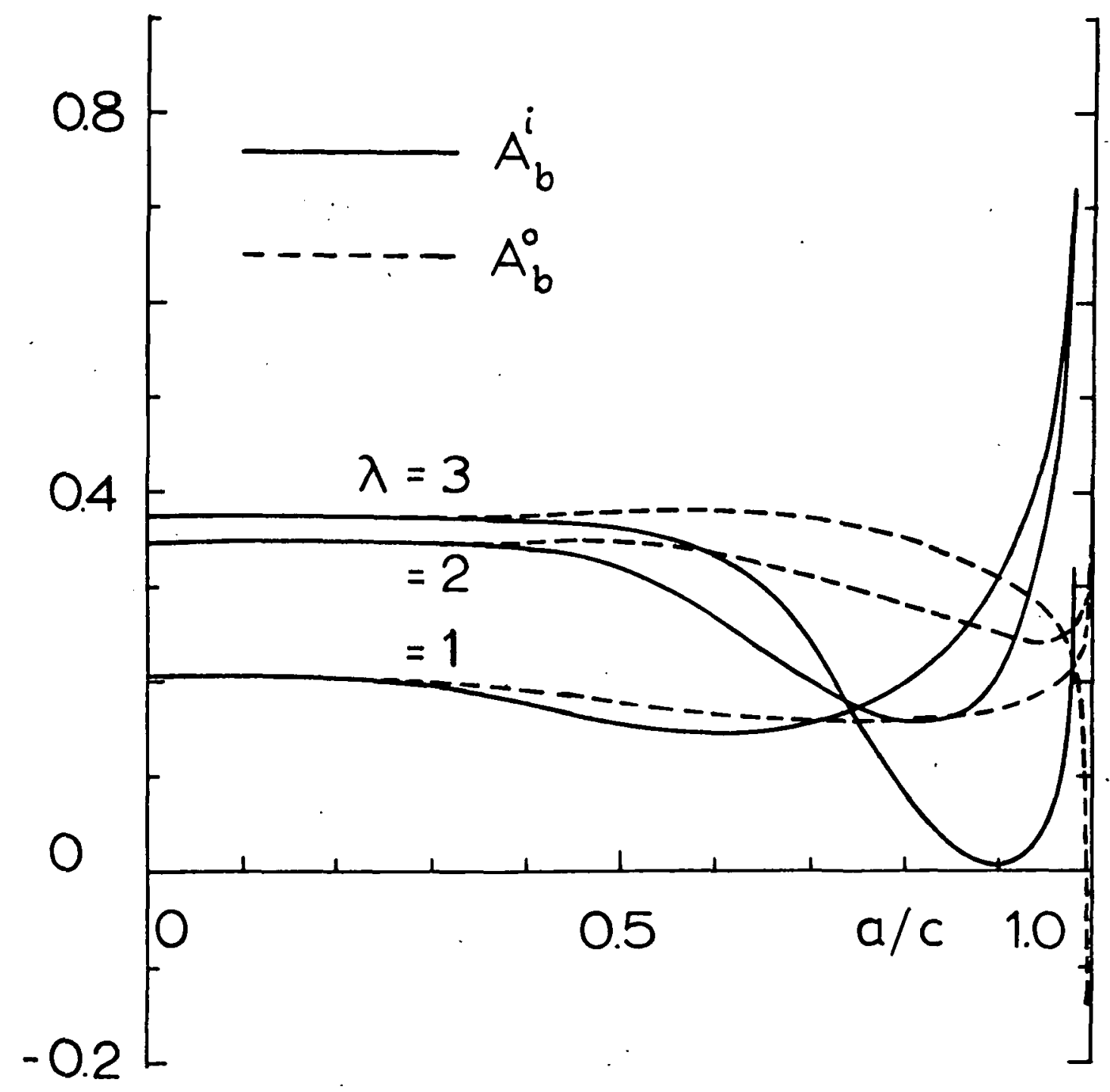

Figure 2. The bending components of the stress intensity factor ratio in a pressurized cylindrical shell with two collinear cracks, $A_{b}^{i}$ for the inner crack tip and $A_{b}^{0}$ for the outer crack tip. 